\title{
A Three-Step Method for Laparoscopic Mobilization of the Splenic Flexure
}

\author{
Takeru Matsuda, $\mathrm{MD}^{1}$, Takeshi Iwasaki, $\mathrm{MD}^{1}$, Kenro Hirata, $\mathrm{MD}^{1}$, Daisuke Tsugawa, $\mathrm{MD}^{1}$, Yutaka Sugita, $\mathrm{MD}^{\mathbf{1}}$, \\ Yasuo Sumi, $\mathbf{M D}^{2}$, and Yoshihiro Kakeji, $\mathbf{M D}^{2}$ \\ ${ }^{1}$ Department of Surgery, National Hospital Organization Kobe Medical Center, Kobe, Japan; ${ }^{2}$ Division of Gastrointestinal \\ Surgery, Department of Surgery, Kobe University Graduate School of Medicine, Kobe, Japan
}

\begin{abstract}
Background. Splenic flexure mobilization (SFM) is sometimes required for secure and tension-free anastomosis during laparoscopic colorectal surgery. Although several approaches have been used for laparoscopic SFM, ${ }^{1}$ it is still considered complicated and troublesome because of the lack of a standardized technique. We propose a three-step method for laparoscopic SFM, aimed at providing a simplified and standardized method, which is presented in the video.

Method. First, the mesocolon of the transverse and descending colon is dissected from the retroperitoneal tissue using a medial approach. The appropriate plane for dissection can be recognized just beneath the inferior mesenteric vein, and the gauze should be placed in the dissected space. Second, the transverse colon and transverse mesocolon are detached from the pancreas and spleen by means of an anterior approach. The lesser sac is opened, and the attachment to the pancreas or spleen is cut using the inserted gauze as a landmark. Third, the lateral attachment of the descending colon to the left abdominal wall is dissected cranially or caudally.
\end{abstract}

Electronic supplementary material The online version of this article (doi:10.1245/s10434-015-4637-6) contains supplementary material, which is available to authorized users.

(C) Society of Surgical Oncology 2015

First Received: 31 March 2015;

Published Online: 29 May 2015

T. Matsuda, MD

e-mail: takerumatsuda@nifty.com
Results. Using this method, we performed laparoscopic SFM for 13 patients with colorectal cancer with no conversion to another approach or to open surgery. No intraoperative complications were reported, including bleeding from the spleen and injury of the pancreas. The mean time for SFM was $55 \mathrm{~min}$.

Conclusions. We consider this three-step method as being useful, and it might help the standardization of laparoscopic SFM.

DISCLOSURE Takeru Matsuda, Takeshi Iwasaki, Kenro Hirata, Daisuke Tsugawa, Yutaka Sugita, Yasuo Sumi, and Yoshihiro Kakeji have no conflicts of interest or financial ties to disclose.

\section{REFERENCE}

1. Benseler V, Hornung M, Iesalnieks I, von Breitenbuch P, Glockzin G, Schlitt HJ, et al. Different approaches for complete mobilization of the splenic flexure during laparoscopic rectal cancer resection. Int J Colorectal Dis. 2012;27(11):1521-9. 